\title{
PREDICTORS OF DRIVING IN INDIVIDUALS WITH RELAPSING-REMITTING MULTIPLE SCLEROSIS
}

Abiodun E. Akinwuntan, Kelly Baker, Michelle Manley, Erin McGonegal, Christina O’Connor, Kendra Phillips \& Kristen Turchi

Georgia Regents University

Augusta, Georgia, USA

Email: aakinwuntan@gru.edu

Summary: Evaluations on fitness-to-drive of individuals with multiple sclerosis (MS) usually involve the administration of several physical, visual, and cognitive tests. In some instances, a practical road test is also administered. The use of several tests, many of which are only remotely driving-related, increases the time, cost, and human resources involved in the evaluation process, and sometimes lead to erroneous decisions.

In this study, we investigated the usefulness of using a short battery of a few highly predictive tests to predict fitness-to-drive of individuals with MS. Fortyfour individuals with relapsing-remitting MS (age = $46 \pm 11$ years, 37 females) and Expanded Disability Status Scale values between 1 and 7 were administered selected physical, visual and cognitive tests including the Stroke Driver Screening Assessment (SDSA) battery.

Performance on 12 cognitive and three visual tests were significantly associated with participants' performance on a practical road test. The Stroop Color test, Direction, Compass, and Road Sign Recognition tests from the SDSA, and the Speed of Processing test from Useful Field of View test battery together explained $59 \%$ of the variance and predicted the pass or fail outcome on the road test with 91\% accuracy, $70 \%$ sensitivity, and $97 \%$ specificity.

The five psychometric/off-road tests, which together can be administered in less than 45 minutes, cost approximately $\$ 150$, and is $91 \%$ accurate, can be used as a screening battery. Those who pass should be further tested on-road to finally decide their fitness-to-drive while those of fail should be further evaluated, trained, or advised on alternative transportation means.

Future studies are needed to confirm and validate the findings in this study.

\section{ACKNOWLEDGMENTS}

This study was supported by a grant from the Consortium of Multiple Sclerosis Centers. 